\title{
Space-Charge Effects on Beam Rotation at the Compressor Ring
}

\author{
K.Y. Ng \\ Fermi National Accelerator Laboratory, Batavia, IL 60510
}

(February 1, 2013)

\begin{abstract}
Bunch-width compression can be accomplished by rf rotating an elongated bunch with minimal energy spread. The longitudinal space-charge force counteracts the rf force. When it is larger than the rf force, the focusing effect of the rf will be lost completely. This possibility is studied with analytic formulas as well as simulations. The transverse space-charge force can drive quadrupole breathing modes in the beam. If the frequencies of these modes fall into the stopbands of parametric resonances, especially the half-integer resonances, emittances will increase. Application is made to the bunch-width compression in the Fermilab Compressor Ring proposed by Alexahin and Neuffer [1], destined for pion and subsequently muon production.
\end{abstract}




\section{Introduction}

The proton source for the muon collider or neutral factory at Fermilab consists of accumulating four bunches each of intensity $N_{b}=0.525 \times 10^{14}$ in the Accumulator Ring (AR) using the $3.87-\mathrm{MHz}$ rf [1]. They are compressed to a final rms fractional energy spread of $\sigma_{E}=5.2 \times 10^{-4}$ and rms bunch length $\sigma_{\tau i}=29.2 \mathrm{~ns}$ by lowering the rf voltage adiabatically. These four bunches are then transferred to the Compressor Ring (CR) one at a time for the neutrino factory or all at the same time for the muon collider. Inside the CR, these bunches are rotated by the $\mathrm{CR} 3.87-\mathrm{MHz}$ rf with a voltage of $V_{\mathrm{rf}}=240 \mathrm{kV}$ and harmonic $h=4$ hoping to create bunches as narrow as rms width $\sigma_{\tau f}=3.2 \mathrm{~ns}$. The question to answer here is whether the space-charge effects of these intense narrow bunches are significant or not. If yes, would they cause any adverse effects to the bunch rotation?

In section 2, we study the longitudinal space-charge effects. We first assume that the linear beam distribution is Gaussian turn after turn. The space-charge induced defocusing force is estimated and compared with the bunching rf force. Then numerical simulations are described by computing the longitudinal space-charge force numerically. Our computation shows that at the present bunch intensity the space-charge distortion of the rf potential, although not small, is still insignificant in causing disastrous effects to the beam narrowing. In section 3, the transverse space-charge forces are computed and the transverse incoherent space-charge tune shifts are derived. Since the coherent dipole space-charge tune shifts vanish, we need to compute the coherent quadrupole coherent space-charge tune shifts of the breathing modes. This requires the solution of the envelope equation. Our computation shows that the coherent quadrupole coherent space-charge tune shifts are $\sim 2 \times 0.06$ horizontally and vertically, which will not be large enough to shift the betatron tunes into the half-integer stopbands. Conclusions are given in Sec. 4.

\section{Longitudinal Space-Charge Effects During Rotation}

\subsection{Estimation}

Consider a bunch of longitudinal linear distribution $\lambda(\tau)$, normalized to unity when integrated over the time advance $\tau$. Due to the variation of the linear distribution, a particle in 
the bunch at the beam axis sees a longitudinal space-charge force

$$
e \mathcal{E}_{\mathrm{spch}}(\tau)=\frac{e^{2} g_{0} N_{b} Z_{0}}{4 \pi \gamma^{2} \beta^{2} c} \frac{d \lambda(\tau)}{d \tau},
$$

where $N_{b}$ is the number of particles in the bunch, $e$ is the proton charge,

$$
g_{0}=1+2 \ln \frac{b}{a}
$$

is the geometric factor, with $a$ and $b$ being the effective beam radius and effective vacuumchamber radius, $\gamma$ and $\beta$ are the relativistic factors, $c$ is the velocity of light, and $Z_{0} \approx 376.6 \Omega$ is the free-space impedance. In one turn, the particle at time-advance $\tau$ gains an energy of $\Delta E_{\mathrm{spch}}=2 \pi \operatorname{Re} \mathcal{E}_{\mathrm{spch}}(\tau)$.

For a Gaussian bunch of rms length $\sigma_{\tau}$, the linear density is

$$
\lambda(\tau)=\frac{1}{\sqrt{2 \pi} \sigma_{\tau}} e^{-\left(\tau-\tau_{0}\right)^{2} / 2 \sigma_{\tau}^{2}},
$$

where $\tau_{0}$ is the time-advance of the center of the bunch. The gradient of the linear density is

$$
\frac{d \lambda(\tau)}{d \tau}=-\frac{\tau-\tau_{0}}{\sqrt{2 \pi} \sigma_{\tau}^{3}} e^{-\left(\tau-\tau_{0}\right)^{2} / 2 \sigma_{\tau}^{2}}
$$

Thus the simplest approximate way to incorporate longitudinal space-charge is to assume the bunch to have a Gaussian distribution at the end of turn, compute the $\sigma_{\tau}$, and use the above expression to compute the increase in energy due to the space-charge force. This energy increase is to be compared with the energy increase coming from the rf voltage wave

$$
\Delta E_{\mathrm{rf}}=e V_{\mathrm{rf}} \sin \frac{2 \pi \tau}{\tau_{b}}
$$

where $\tau_{b}=T_{0} / h$ is the bucket width at the $\mathrm{rf}$ harmonic $h, T_{0}=2 \pi / \omega_{0}$ is the revolution period of the ring, and $V_{\mathrm{rf}}$ is the rf voltage. The ratio of the two energy increases is

$$
\mathcal{R}=\frac{\Delta E_{\mathrm{spch}}}{\Delta E_{\mathrm{rf}}}
$$

Using the expressions in Eqs. (2.1), (2.4), (2.5), and limiting the derivation to region close to the longitudinal center of the bunch, we obtain

$$
\mathcal{R}=\frac{1}{2(2 \pi)^{5 / 2}} \frac{e g_{0} N_{b} Z_{0} T_{0}^{2}}{\gamma^{2} \beta \sigma_{\tau}^{3} V_{\mathrm{rf}} h} .
$$

Actually, $\mathcal{R}$ denotes the ratio of the gradients of the two forces. Now the CR parameters listed in Table I are substituted [1]. With the space-charge geometric parameter $g_{0}=5$ and 
Table I: Some parameters of the CR and its bunches

Circumference (m)

308.23

Momentum compaction

0.0010

Kinetic beam energy $(\mathrm{GeV})$

Revolution frequency $f_{0}(\mathrm{MHz})$

$\mathrm{RF}$ harmonic $h$

$\mathrm{RF}$ voltage $(\mathrm{kV})$

Slippage factor $\eta$ $-1.002 \times 10^{-2}$

Synchrotron tune $\nu_{s}$ $4.161 \times 10^{-4}$

Initial rms bunch width $\sigma_{\tau i}$ (ns)

Initial rms energy spread $\Delta E / E$

$5.2 \times 10^{-4}$

Rms unnormalized vertical/horizontal emittance $\epsilon_{y, x}(\pi \mu \mathrm{m})$

Betatron bare tunes $\nu_{0 y} / \nu_{0 x}$

$6.76 / 8.44$

final rms beam width $\sigma_{\tau f}=3.2 \mathrm{~ns}$ at the end of rotation, this space-charge-to-rf-force ratio is $\mathcal{R}=29.5$. In other words, the space-charge force dominates over the rf force at the center of the rotated beam, and beam rotation can become impossible. To draw a conclusion, however, we must recognize the followings:

1. The final rms bunch length of $\sigma_{\tau f}=3.2 \mathrm{~ns}$ quoted in Ref. [1] probably comes from extracting the central part of the rotated bunch. There are tails on both sides of the bunch because the initial bunch before rotation is of rms width $\sigma_{\tau i}=29.2 \mathrm{~ns}$, which is quite long. The rf bucket width is $\tau_{b}=258.46 \mathrm{~ns}$. The space-charge force is proportional to the gradient of the beam including the tails. Thus in Eq. (2.7), the rms width of the whole beam should be used instead. In Fig. 2 below, simulation shows that the rms width of the whole beam after rotation is $\sigma_{\tau f} \sim 5.0 \mathrm{~ns}$. Using this ratio, the space-charge force ratio reduces to $\mathcal{R}=7.73$, which is 3.8 times smaller.

2. During bunch rotation, the bunch width contracts and assumes a minimum only at the end of the rotation. The force ratio, according to above, is

$$
\mathcal{R} \propto \frac{1}{\sigma_{\tau}^{3}}=\frac{1}{\left(\sigma_{\tau f}^{2} \sin ^{2} \omega_{s} t+\sigma_{\tau i}^{2} \cos ^{2} \omega_{s} t\right)^{3 / 2}} .
$$

The variation of the space-charge force ratio $\mathcal{R}$ during the rotation process is depicted in Fig. 1. We see that the ratio increases from $\mathcal{R}=0.0388$ at start to 7.727 at the end 


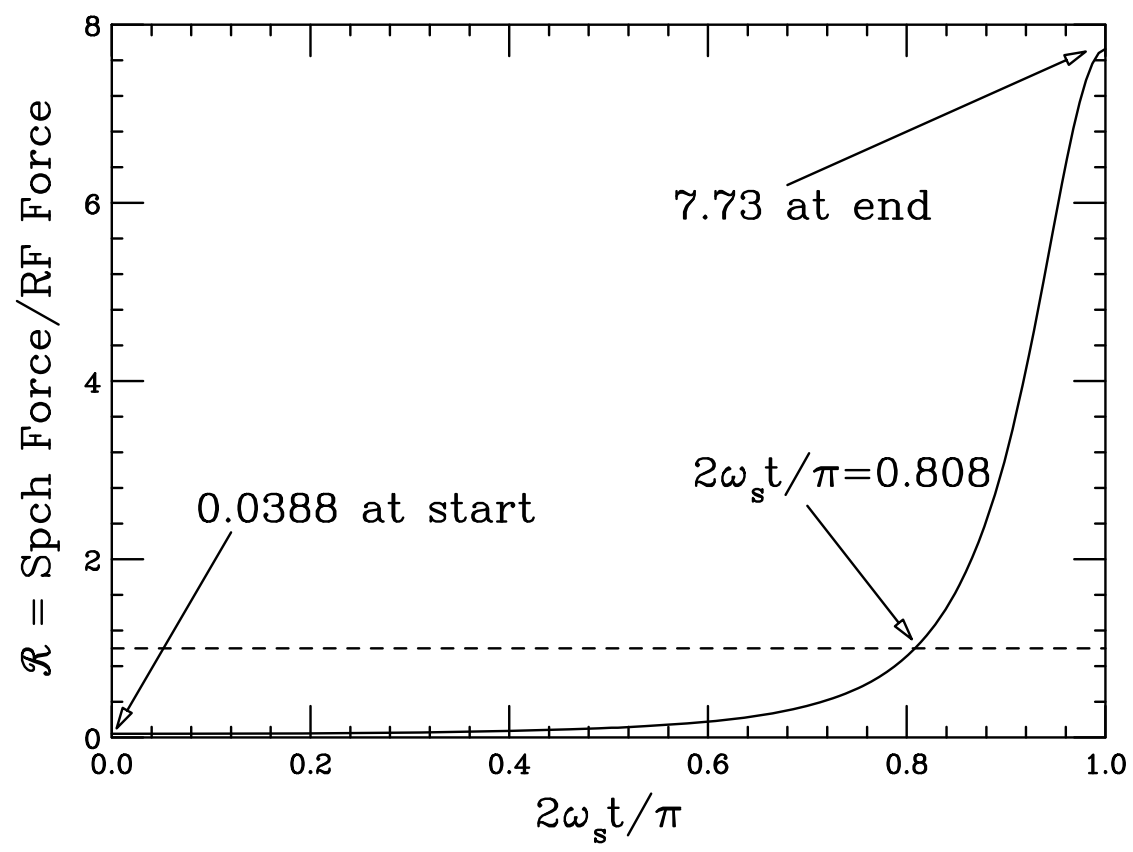

Figure 1: The ratio $\mathcal{R}$ of the space-charge force to the rf force as a function of time is shown. The ratio increases from $\mathcal{R}=0.0388$ at start to 7.727 at the end of the rotation.

of the rotation. Note that $\mathcal{R}>1$ and increases sharply for only $20 \%$ of the time near the end of the rotation. The small-amplitude synchrotron tune is $\nu_{s}=4.16 \times 10^{-4}$, or the rotation takes place for about 600 turns. Thus the total force, space-charge plus rf, is defocusing at the center of the bunch for about 120 turns only. It is hard to determine whether how badly the bunch shape will be distorted and how long the bunch will be lengthened.

\subsection{Numerical Gradient Computation}

The more accurate method, of course, is to compute the gradient of the beam density numerically. First divide the bucket into $n$ bins each of width $\Delta \tau=\tau_{b} / n$. Next the time advance $\tau_{i}$ of the $i^{\text {th }}$ particle is translated to $S_{i}$, which ranges from 0 to $n$ from the right side of the bucket to the left, or

$$
S_{i}=\tau_{i} \frac{n}{\tau_{b}}
$$

In this way, this particle will be placed into the $k_{i}^{\text {th }}$ bin, where

$$
k_{i}=\operatorname{int}\left(S_{i}\right)+1
$$


with $\operatorname{int}\left(S_{i}\right)$ denoting the integer part of $S_{i}$. After the placement into bins, the number of particles in the $k^{\text {th }}$ bin is $m_{k}$, with $\sum_{k} m_{k}=N_{b}$. The linear distribution is now

$$
g_{k}=\frac{m_{k}}{N_{b}}
$$

with $\sum_{k} g_{k}=1$. Now define for each particle

$$
s_{i}=S_{i}-\left(k_{i}-\frac{1}{2}\right)
$$

where $s_{i}$ is measured with respect to $k_{i}-\frac{1}{2}$, which is the center of the $k_{i}^{\text {th }}$ bin where $i^{\text {th }}$ particle resides. This definition leads to $\left|s_{i}\right|<\frac{1}{2}$. The contribution of this particle to the gradient of the linear distribution at $k_{i}^{\text {th }}$ bin is, according to the three-point differentiation rule $[2]$,

$$
\frac{d \lambda}{d \tau}=\frac{d g_{k_{i}}}{d \tau}=\frac{1}{\tau_{b}^{2}} \sum_{\substack{\text { all in } \\ \text { bin } k_{i}}}\left[\left(s_{i}-\frac{1}{2}\right) g_{k_{i}-1}-2 s_{i} g_{k_{i}}+\left(s_{i}+\frac{1}{2}\right) g_{k_{i}+1}\right],
$$

where the summation over $i$ is over all particles in the $k_{i}^{\text {th }}$ bin. In above, $\lambda(\tau)$ is the linear distribution, which is normalized to unity when integrated over $\tau$. Thus

$$
\lambda(\tau) d \tau=g_{k} d k \quad \text { or } \quad \lambda(\tau)=g_{k} \frac{d k}{d \tau}=\frac{g_{k}}{\Delta \tau} .
$$

\subsection{Simulations}

The width of the rf bucket in the $\mathrm{CR}$ is $\tau_{b}=258.5 \mathrm{~ns}$. We divide it into $n=100$ equal bins, so that the bin width is $\Delta \tau=\tau_{b} / n=2.585 \mathrm{~ns}$. The rotated beam is expected to have an $\mathrm{rms}$ width of $\sigma_{\tau} \sim 3 \mathrm{~ns}$. Thus the beam occupies about 5.7 bins. The beam at start of rotation is populated randomly in the longitudinal phase space in a bi-Gaussian way with $\sigma_{\tau i}=29.2 \mathrm{~ns}$ and fraction energy offset $\sigma_{E i}=5.20 \times 10^{-4}$. One million macroparticles are employed in each simulation, so that there will on the average 10000 macroparticles in each bin. The statistical error will be $\sim 1 \%$, and will be much smaller in the central part of the beam. So many macroparticles are needed in each bin because the linear density gradient is to be computed. We consider this choice of binning parameters is reasonable in the simulations. To support this claim, we have tried (1) to increase the number of bins to 150, and (2) to decrease the number of macroparticles by a factor of two. There are no significant changes in the outcome. 

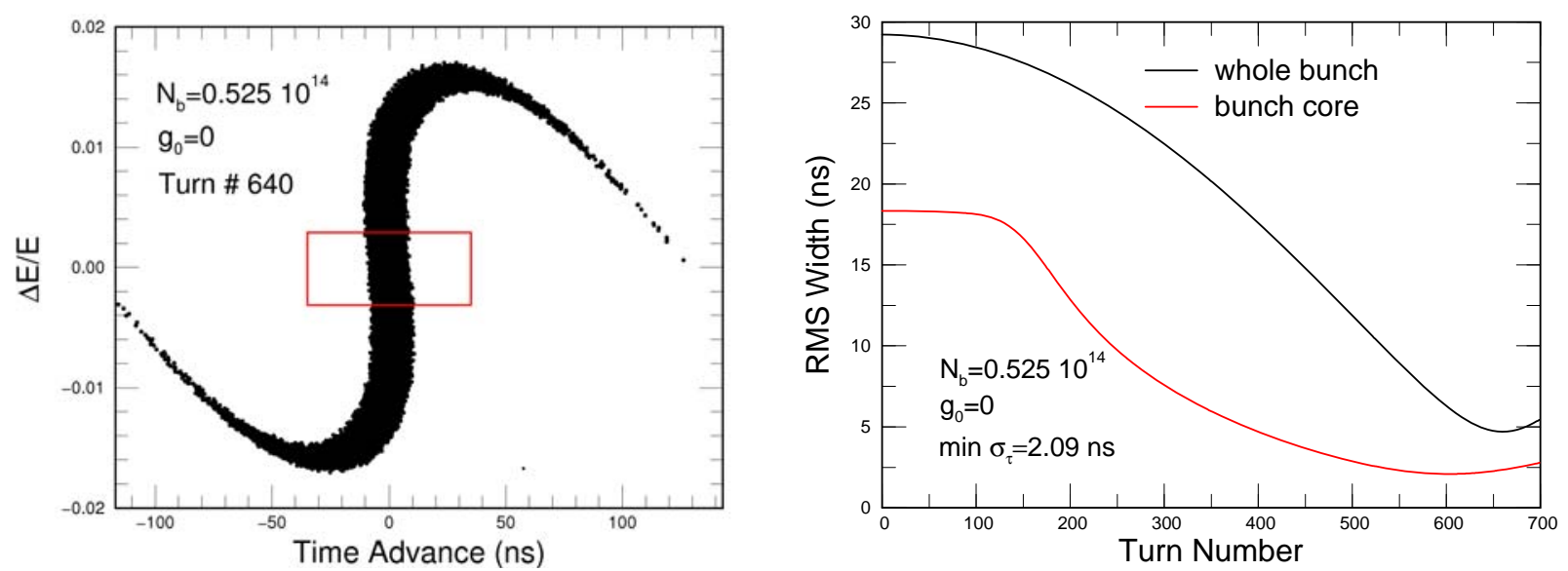

Figure 2: Left: Rotated bunch in the longitudinal phase space with space-charge turned off $\left(g_{0}=\right.$ $0)$. One million macroparticles are used. Right: Beam rms width $\sigma_{\tau}$ versus rotation turn number is shown in black when all beam particles are taken into account, and is shown in red when only an energy-offset portion (depicted as the red box on the left plot) is considered.

The first simulation depicted in Fig. 2 is for the situation when space-charge is turned off $\left(g_{0}=0\right)$. The left plot shows the particle distribution in the longitudinal phase space after rotation, at turn 640. The rms bunch length at each turn is shown in black in the right plot. The minimum is $4.73 \mathrm{~ns}$ at turn 660 . This computation of the rms bunch length includes the tails on both sides of the beam core. Also shown in the plot is the rms width of beam core, which is defined here as energy offset between $\Delta E / E= \pm 0.003$ and time advance between $\tau= \pm 35 \mathrm{~ns}$, depicted as the red box in the left plot of Fig. 2. The minimum rms bunch width is $2.09 \mathrm{~ns}$ at turn 601 . This value can be different from the rms beam width extracted, for example, the value quoted by Ref. [1] in Table I. However, it serves here as a measure of how narrow the beam core becomes after rotation. For the initial rms fractional energy offset $\sigma_{E i}=5.20 \times 10^{-4}$, the bunch rotated by an rf voltage of $240 \mathrm{kV}$ should have an rms width of $\sigma_{\tau f}=|\eta| \sigma_{E i} /\left(\beta^{2} \omega_{s}\right)=2.08 \mathrm{~ns}$, where we have used synchrotron frequency $\omega_{s} / 2 \pi=\nu_{s} f_{0}$, with synchrotron tune $\nu_{s}=4.162 \times 10^{-4}$, revolution frequency $f_{0}=0.9673 \mathrm{MHz}$, and slip factor $\eta=-1.0019 \times 10^{-2}$. The agreement with simulation has been remarkable.

Now space-charge is turned on. The left and right plots in Fig. 3 are simulations using space-charge geometric factors $g_{0}=2.5$ and 5 , respectively. The total voltage, space-charge and rf, in the middle plots show defocusing effects at the bunch center at turn 640. Compared with the phase-space plot in Fig. 2, we can see from the first row of Fig. 3 a slight distortion of the beam shape coming from space-charge when $g_{0}=2.5$ and a more pronounced distortion 

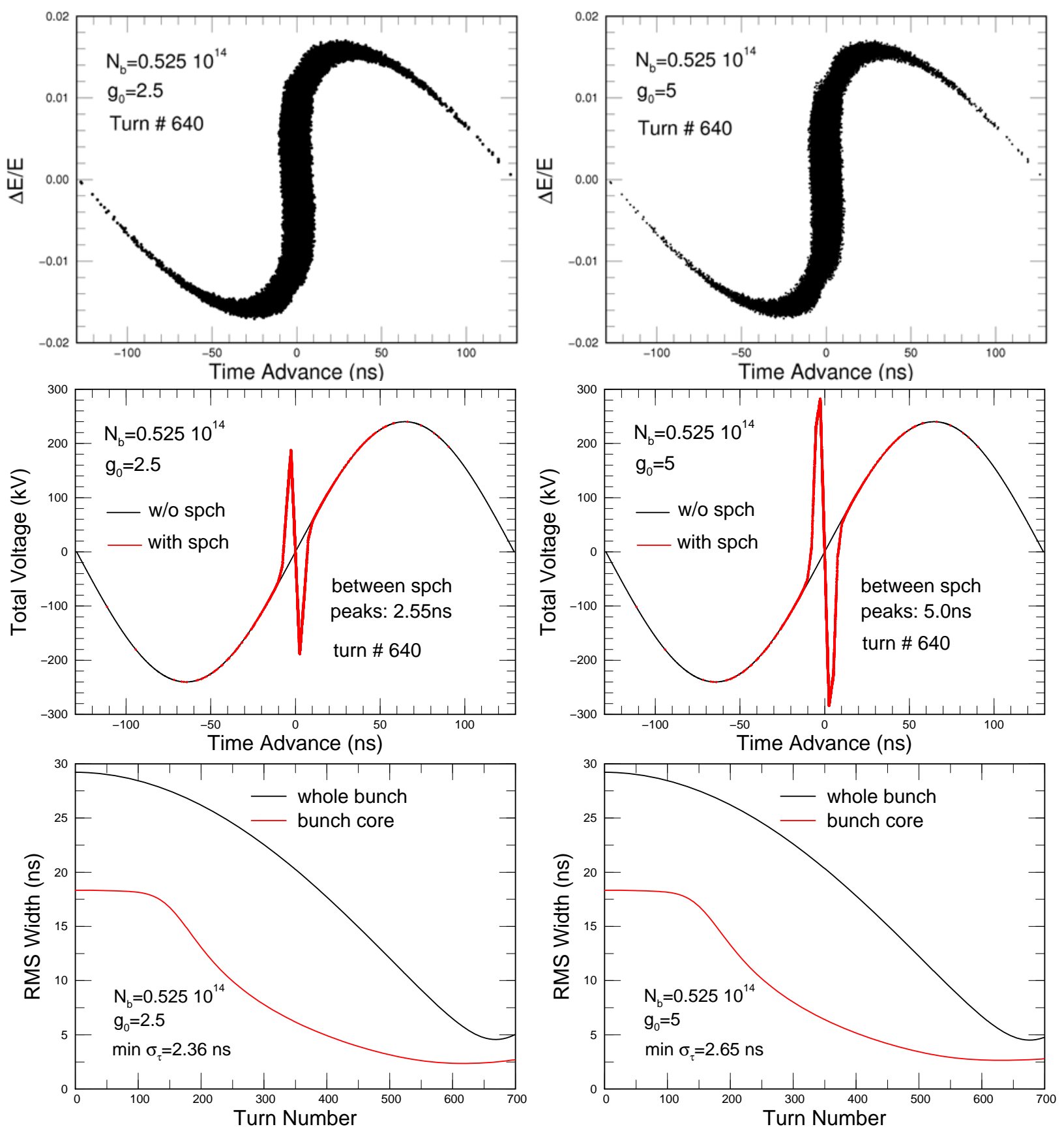

Figure 3: Left column is for space-charge geometric parameter $g_{0}=2.5$ and right column is for $g_{0}=5$. First row: Rotated bunch in the longitudinal phase space. One million macroparticles are used. Second row: Total voltage in red, space-charge and rf, encountered by beam particles at turn 640. The rf voltage is in black. Third row: Beam rms width $\sigma_{\tau}$ versus rotation turn number is shown in black when all beam particles are taken into account, and is shown in red when only the beam core, $|\Delta E / E|<0.003$ and $|\tau|<35$ ns, is included. 
when $g=5$. The rms width of the core grows to $2.36 \mathrm{~ns}$ when $g_{0}=2.5$ and $2.65 \mathrm{~ns}$ when $g_{0}=5$. Notice that in Eq. (2.1), for example, $g_{0} N_{b}$ goes together. In other words, increasing $g_{0}$ is the same as increasing the beam intensity.

Figure 4 is the same as Fig. 3, but with the space-charge geometrical parameter increases to $g_{0}=10$ and $g_{0}=20$. Now the effects of the space-charge force are very much more pronounced. The rms bunch width after rotation increases to $\sigma_{\tau f}=3.24$ and $4.49 \mathrm{~ns}$, respectively.

\subsection{Comments}

The space-charge geometrical parameter $g_{0}$ is usually between 3 and 5 . Even if $g_{0}=5$, for the present beam intensity, the longitudinal space-charge does plays some role in the bunch rotation. In the presence of space-charge, the rms width of the beam core increases by $(2.65-2.09) / 2.09=27 \%$. In the future, if the beam intensity is doubled $\left(g_{0}=5\right)$, the increase in bunch width will increase by $(3.24-2.09) / 2.09=55 \%$. A quadruple increase in beam intensity will have the rotated bunch width more than doubled. The rms widths of the bunch core, defined by $|\Delta E / E|<0.003$ and $|\Delta \tau|<35$ ns, for various space-charge factor $g_{0}$ are shown in Fig. 5 .

\section{Transverse Space-Charge Effects}

As the bunch narrowing proceeds, the space-charge tune shifts increase. We wish to investigate whether the changes in tune shifts will be so large that the beam encounters the integer stopband, half-integer stopband, or some important nonlinear resonances, resulting in the increase of the transverse emittances.

\subsection{Inocherent Space-Charge Tune Shifts}

The horizontal and vertical space-charge tune shifts at the center of the beam are

$$
\Delta \nu_{x, y}^{\mathrm{spch}}=\frac{N_{b} r_{0} R}{2 \pi \gamma^{3} \beta^{2} \nu_{x, y}}\left\langle\frac{1}{\sigma_{x, y}\left(\sigma_{x}+\sigma_{y}\right)}\right\rangle \frac{I_{\mathrm{pk}}}{I_{\mathrm{av}}},
$$



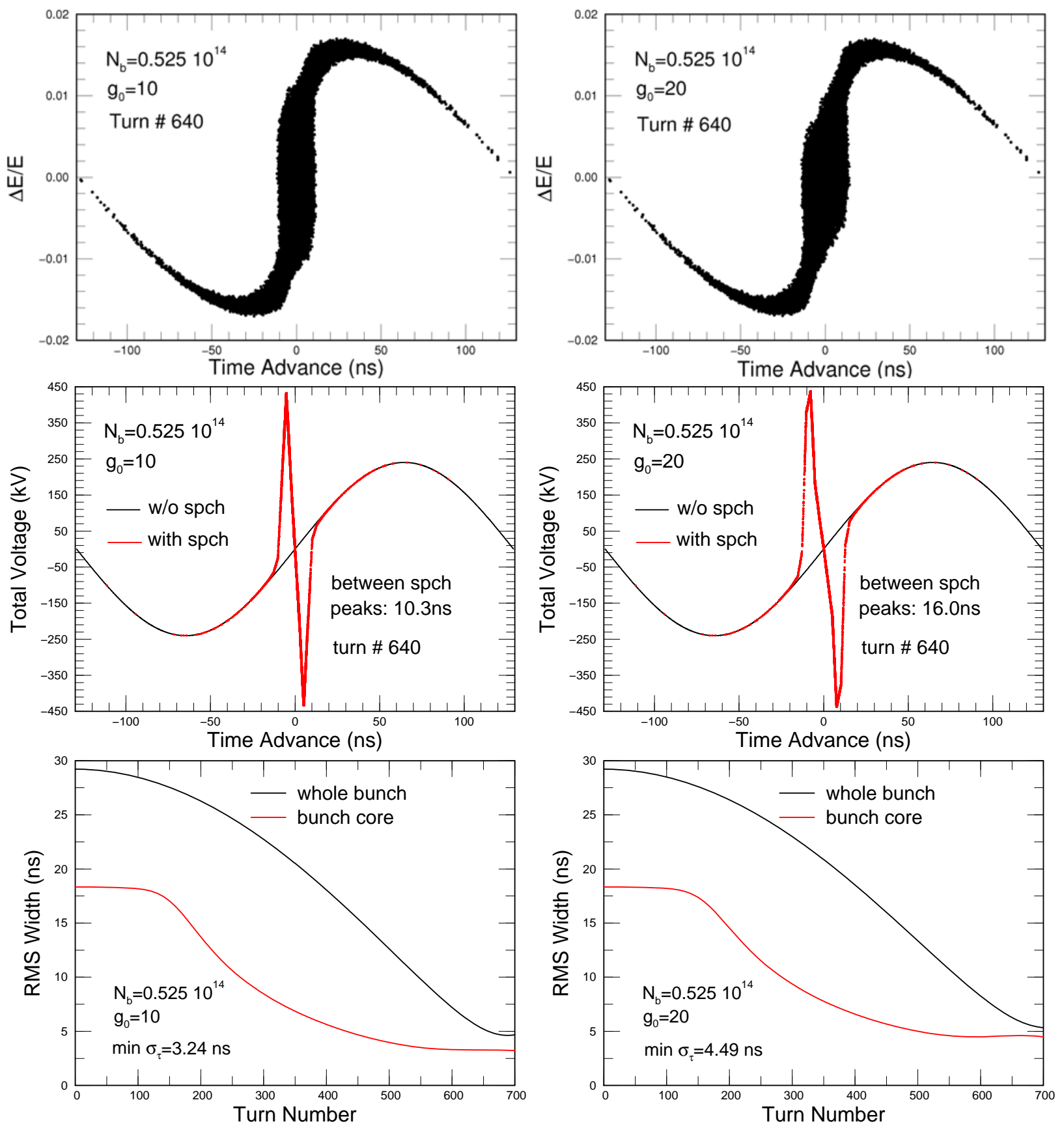

Figure 4: Left column is for space-charge geometric parameter $g_{0}=10$ and right column is for $g_{0}=20$. First row: Rotated bunch in the longitudinal phase space. One million macroparticles are used. Second row: Total voltage in red, space-charge and rf, encountered by beam particles at turn 640. The rf voltage is in black. Third row: Beam rms width $\sigma_{\tau}$ versus rotation turn number is shown in black when all beam particles are taken into account, and is shown in red when only the beam core, $|\Delta E / E|<0.003$ and $|\tau|<35 \mathrm{~ns}$, is included. 


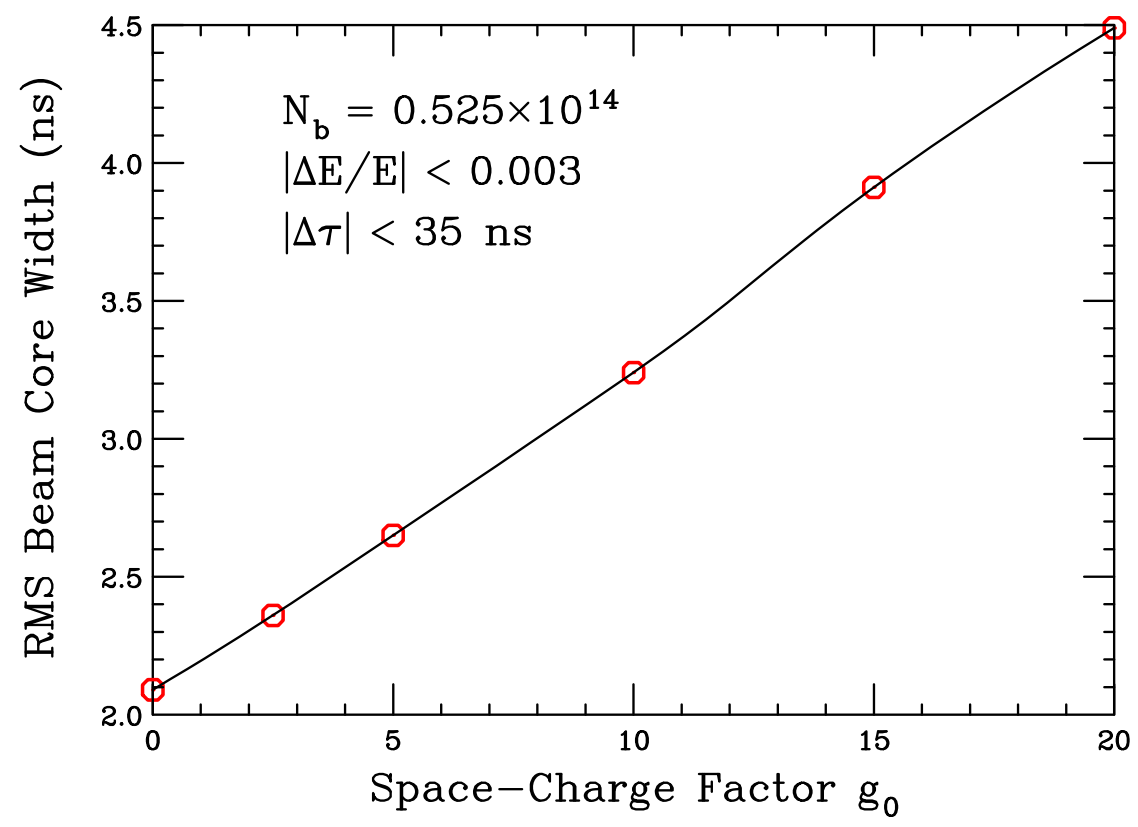

Figure 5: Rms width of the bunch core after rotation at various space-charge geometrical factor $g_{0}$, but with bunch intensity fixed at $N_{b}=0.525 \times 10^{14}$. The bunch core is defined as $|\Delta E / E|<0.003$ and $|\Delta \tau|<35 \mathrm{~ns}$, depicted as the red box in the left plot of Fig. 2.

where $I_{\mathrm{pk}}$ and $I_{\mathrm{av}}$ are the peak and average beam current,

$$
r_{0}=\frac{e^{2}}{4 \pi \epsilon_{o} m c^{2}}=\frac{e^{2} Z_{0} c}{4 \pi m_{p} c^{2}}
$$

is the classical proton radius, and $m_{p} c^{2}$ is the proton rest energy. The horizontal and vertical rms beam radii are denoted by $\sigma_{x, y}$ and $\langle\cdots\rangle$ implies the averaging around the accelerator ring. Since the CR is composed of an ordinary FODO lattice without exotic low-beta and/or high-beta regions, we may employ the smooth approach by using average betatron functions

$$
\bar{\beta}_{x, y}=\frac{R}{\nu_{x, y}} .
$$

and average rms beam radii. The average vertical $\mathrm{rms}$ beam radius is given by

$$
\sigma_{y}=\sqrt{\bar{\beta}_{y} \epsilon_{y}}
$$

where $\epsilon_{x, y}$ denote the unnormalized rms horizontal and vertical emittances of the beam. Since the horizontal beam size receives contribution also from the dispersion, the average horizontal rms beam radius takes the form

$$
\sigma_{x}=\sqrt{\bar{\beta}_{x} \epsilon_{x}+\left(\sigma_{E} \bar{D}\right)^{2}}
$$




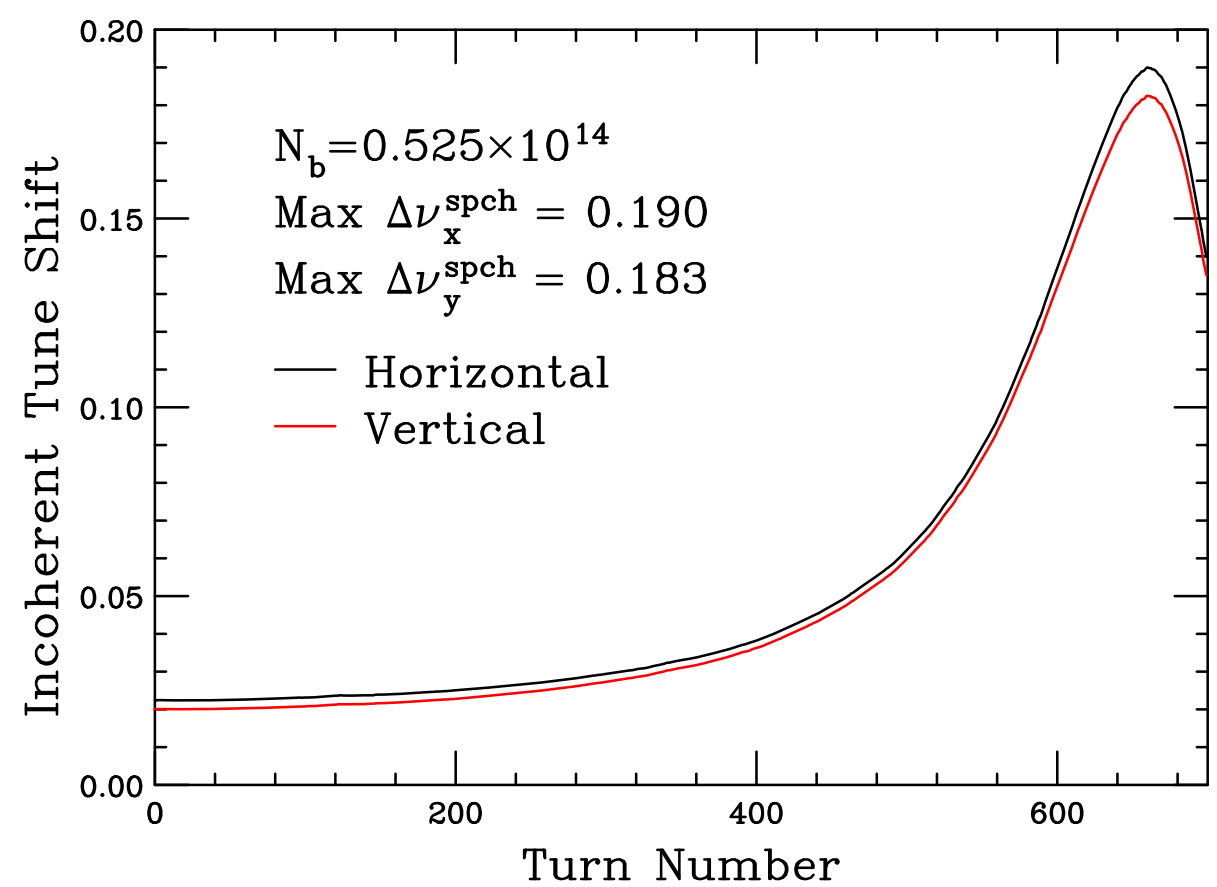

Figure 6: Maximum space-charge tune shifts of the beam in the CR during rotation with bunch intensity $N_{b}=0.525 \times 10^{14}$.

where $\sigma_{E}$ is the rms momentum spread and $\bar{D}$ is the average dispersion, which is approximately $0.4 \mathrm{~m}$.

For the beam injected into the $\mathrm{CR}$, the unnormalized horizontal and vertical rms emittances are both equal to $\epsilon_{x, y}=5 \pi \mu \mathrm{m}$. The maximum space-charge tune shift occurs in the time slice where the local current peaks. The space-charge tune shifts are at maximum at turn 660. At that moment the rms energy spread is at a maximum of $\sigma_{E}=5.86 \times 10^{-3}$. The transverse beam sizes are $\sigma_{x}=6.46 \mathrm{~mm}$ and $\sigma_{y}=5.39 \mathrm{~mm}$. For bunch intensity $N_{b}=0.525 \times 10^{14}$, the evolution of the incoherent space-charge tune shifts during rotation are shown in Fig. 6. The maxima are $\Delta \nu_{x}^{\text {spch }}=0.190$ and $\Delta \nu_{y}^{\text {spch }}=0.183$.

The numerical results in Fig. 6 are different from $\Delta \nu_{x, y}^{\text {spch }}=0.14 / 0.16$ quoted in Ref. [1]. The first difference is the rather lower tune-shift values than the simulation made in Fig. 6 . This may come about because we have been smoothing the CR lattice by introducing average beam radii and average betatron functions. The second difference is the smaller horizontal tune shift than the vertical. Notice that the incoherent space-charge tune shifts scale as

$$
\frac{\Delta \nu_{x}^{\text {spch }}}{\Delta \nu_{y}^{\text {spch }}}=\frac{\nu_{0 y} \sigma_{y}}{\nu_{0 x} \sigma_{x}},
$$


where $\nu_{0 x, 0 y}$ are the bare betatron tunes. If the dispersion is not included, this becomes

$$
\frac{\Delta \nu_{x}^{\text {spch }}}{\Delta \nu_{y}^{\text {spch }}}=\sqrt{\frac{\nu_{0 y} \epsilon_{y}}{\nu_{0 x} \epsilon_{x}}}
$$

Thus when the horizontal and vertical emittances are equal, larger betatron tune implies smaller incoherent space-charge tune shift. The horizontal and vertical bare betatron tunes are quoted in Ref. [1] as $\nu_{0 x}=6.76$ and $\nu_{0 y}=8.44$. Thus the horizontal incoherent spacecharge tune shift should be larger than the vertical. It will be the dispersion function that can change this around. So far we have been using an average dispersion of $\bar{D}=0.4 \mathrm{~m}$, and the horizontal incoherent space-charge tune shift is larger than the vertical. We need to raise it to $0.5 \mathrm{~m}$ to produce equal maximum incoherent space-charge tune shift for the horizontal and vertical, and $\bar{D}=0.6 \mathrm{~m}$ for the vertical to be $\sim 4 \%$ larger than the vertical. But the lattice in Fig. 4 of Ref. [1] does not depict dispersion function of this large size. In the discussion below, however, we will use the results $\Delta \nu_{x, y}^{\text {spch }}=0.14 / 0.16$ quoted in Ref. [1]. This is because we believe that the detailed CR lattice has been taken into account there; the results should be more accurate than our approximate computation here.

\subsubsection{Interpretation}

The maximum space-charge tune shifts are experienced by those particles at the center of the central transverse slice of the beam. The gradient of the transverse space-charge force decreases away from the center of the bunch and reverses sign roughly at one sigma of the beam. If we make a rough model by assuming those particles within one sigma of the beam core to have the maximum tune shifts in all transverse directions, while those outside do not experience any space-charge force, the average space-charge tune shift will be reduced by the factor of $1-e^{-1 / 2}=0.393$ from the maxima to $\left(\Delta \nu_{x}^{\text {spch }}\right)_{\mathrm{av}}=0.055$ and $\left(\Delta \nu_{y}^{\mathrm{spch}}\right)_{\mathrm{av}}=0.063$. This estimation, however, is for the transverse slice where the peak local current or linear density is maximum. We can extend the above model to the longitudinal direction as well; i.e., particles residing in one sigma in the transverse and longitudinal direction will experience the maximum space-charge tune shift, while particles outside one sigma does not experience anything. The fractional particle number within the $3-\mathrm{D}$ one sigma is

$$
\frac{1}{(2 \pi)^{3 / 2}} \int_{0}^{1} e^{-r^{2} / 2} r^{2} d r d \Omega=\operatorname{erf}\left(\frac{1}{2}\right)-\sqrt{\frac{2}{\pi}} e^{-1 / 2}=0.199,
$$

where $\operatorname{erf}(x)$ is the error function. Thus in this model, the average space-charge tune shifts at the end of the bunch rotation are $\left(\Delta \nu_{x}^{\mathrm{spch}}\right)_{\mathrm{av}}=0.028$ and $\left(\Delta \nu_{y}^{\mathrm{spch}}\right)_{\mathrm{av}}=0.032$. What 
we want to point out is that there is not a unique way to quote the incoherent spacecharge tune shifts of a bi-Gaussian or tri-Gaussian distributed bunch. However, it has been shown repeatedly in the literature that the incoherent space-charge tune shifts alone do not determine the encountering of parametric resonances $[3,4,5]$. It is the coherent spacecharge tune shifts that determine when a parametric resonance is encountered. The theory behind this statement is reviewed below. After that the coherent space-charge tune shifts are computed.

\subsection{The Integer and Half-Integer Resonances}

For simplicity, we work in the Floquet space. The Floquet horizontal displacement $X$ and phase $\psi_{x}$ are defined as

$$
X=\frac{x}{\sqrt{\beta_{x}}} \quad \text { and } \quad \psi_{x}=\int_{0}^{s} \frac{d s}{\nu_{0 x} \beta_{x}(s)},
$$

where $x$ is the particle horizontal displacement at location $s$ along the designed closed orbit, $\beta_{x}(s)$ is the horizontal betatron function, and $\nu_{0 x}$ is the bare horizontal betatron tune. The equation of motion governing the $i$ th particle in the horizontal direction is

$$
\frac{d^{2} X_{i}}{d \psi_{x}^{2}}+\nu_{0 x}^{2} X_{i}=\sum_{j}^{\prime} F_{i j}+F_{x}^{\mathrm{ext}}\left(\psi_{x}\right)
$$

where $F_{i j}$ is horizontal component of the force the $j$ th particle acting on the $i^{\text {th }}$ particle, and $\sum_{j}^{\prime}$ implies the summation over $j$ but with $j=i$ excluded. Thus, $\sum_{j}^{\prime} F_{i j}$ is just the horizontal space-charge force from all other particles acting on the $i^{\text {th }}$ particle. The external transverse force $F_{x}^{\text {ext }}\left(\psi_{x}\right)$ comes from lattice error or imperfection around the ring. We now take the average of Eq. (3.24) by summing over $i$, giving exactly

$$
\frac{d^{2}\langle X\rangle}{d \psi_{x}^{2}}+\nu_{0 x}^{2}\langle X\rangle=F_{x}^{\mathrm{ext}}\left(\psi_{x}\right)
$$

This result is obtained because of Newton's third law: $F_{i j}=-F_{j i}$ when $i \neq j$. Subtracting Eq. (3.25) from Eq. (3.24), we arrive at the incoherent equation

$$
\frac{d^{2}}{d \psi_{x}^{2}}(X-\langle X\rangle)+\nu_{0 x}^{2}\left(X_{i}-\langle X\rangle\right)=\sum_{j}^{\prime} F_{i j} .
$$

If the space-charge force $\sum_{j}{ }_{j} F_{i j}$ on the right-hand side is linearized, it will give rise to an incoherent space-charge tune shift for particle $i$. We learn from Eq. (3.25) that the external 
force from the lattice $F_{x}^{\text {ext }}$ will drive the center of the beam into resonance if the bare tune $\nu_{0 x}$ is an integer. However, this external force is absent in Eq. (3.26) and therefore will not drive any integer resonance no matter how big the incoherent space-charge tune shift is.

We try to study the half-integer resonance in a similar way. The equation of transverse motion for a particle with an external quadrupole-error force $X F\left(\psi_{x}\right)$ is

$$
\frac{d^{2} X}{d \psi_{x}^{2}}+\nu_{0 x}^{2} X=-2 \nu_{0 x} \Delta \nu_{x}^{\mathrm{spch}}(X-\langle X\rangle)+X F\left(\psi_{x}\right)
$$

where a linear space-charge force $-2 \nu_{0 x} \Delta \nu_{x}^{\text {spch }}(X-\langle X\rangle)$ has been assumed. Coherent motion is obtained by averaging Eq. (3.27),

$$
\frac{d^{2}\langle X\rangle}{d \psi_{x}^{2}}+\nu_{0 x}^{2}\langle X\rangle=\langle X\rangle F\left(\psi_{x}\right)
$$

and the difference gives the incoherent motion,

$$
\frac{d^{2}}{d \psi_{x}^{2}}(X-\langle X\rangle)+\left(\nu_{0 x}^{2}+2 \nu_{0 x} \Delta \nu_{x}^{\mathrm{spch}}\right)(X-\langle X\rangle)=(X-\langle X\rangle) F\left(\psi_{x}\right) .
$$

Equation (3.28) does show that the external quadrupole-error force acts on the center of the bunch and a half-integer resonance will be encountered if the bare tune $\nu_{0 x}$ is at a half integer. However, Eq. (3.29) shows that the incoherent motion is driven by the quadrupole-error force as well. But we should remember that a quadrupole in the lattice will change the size of the particle beam and so will the quadrupole-error force. The incoherent space-charge tune shift depends on the beam size, which is a function of the quadrupole error force $X F\left(\psi_{x}\right)$. Actually, the effect of the quadrupole-error force inside the incoherent space-charge tune shift just cancels the quadrupole-error force on the right side of Eq. (3.29), leaving behind an incoherent motion not affected by the quadrupole-error force [7].

So far we have shown that the dipole coherent space-charge tune shifts vanish, because the field pattern moves with the beam. Thus to study the transverse space-charge effects on the beam, we need to derive the coherent quadrupole space-charge tune shifts. Since the space-charge force couples the horizontal and vertical phase spaces, we need to go to the two-dimensional envelope equation and study the collective modes of oscillation that are nonrigid. 


\subsection{Sacherer Envelope Equations}

Sacherer [6] showed that the rms horizontal width $\tilde{x}=\sqrt{\left\langle(x-\langle x\rangle)^{2}\right\rangle}$ and rms vertical width $\tilde{y}=\sqrt{\left\langle(y-\langle y\rangle)^{2}\right\rangle}$ of a beam that has the elliptical symmetrical distribution of the form

$$
n(x, y ; s)=n\left(\frac{x^{2}}{a^{2}}+\frac{y^{2}}{b^{2}} ; s\right)
$$

satisfy the two-dimension envelope equation:

$$
\begin{aligned}
& \tilde{x}^{\prime \prime}+K_{x}(s) \tilde{x}-\frac{\epsilon_{x}^{2}}{\tilde{x}^{3}}-\frac{r_{0} \lambda}{\gamma^{3} \beta^{2}} \frac{1}{\tilde{x}+\tilde{y}}=0 \\
& \tilde{y}^{\prime \prime}+K_{y}(s) \tilde{y}-\frac{\epsilon_{y}^{2}}{\tilde{y}^{3}}-\frac{r_{0} \lambda}{\gamma^{3} \beta^{2}} \frac{1}{\tilde{x}+\tilde{y}}=0 .
\end{aligned}
$$

In above, $K_{x}(s)$ and $K_{y}(s)$ are the horizontal and vertical quadrupole force constants,

$$
\epsilon_{x}=\sqrt{\left\langle\Delta x^{2}\right\rangle\left\langle\Delta p_{x}^{2}\right\rangle-\left\langle\Delta x \Delta p_{x}\right\rangle^{2}} \text { and } \epsilon_{y}=\sqrt{\left\langle\Delta y^{2}\right\rangle\left\langle\Delta p_{y}^{2}\right\rangle-\left\langle\Delta y \Delta p_{y}\right\rangle^{2}}
$$

are the horizontal and vertical unnormalized rms emittances, $p_{x, y}$ are the horizontal and vertical canonical momenta, $\lambda$ is the linear density that the distribution $n(x, y ; s)$ is normalized to after integration over $x$ and $y, r_{0}$ is the classical radius of the beam particle. The last terms in Eq. (3.33) are the space-charge forces. The imperfect lattice error around the ring that drive the integer and half-integer resonances has been left out for the time being, but will be added later in Eq. (3.37) below. For a uniform distribution with elliptical symmetry in two dimensions, the half widths of the beam are $\hat{x}=2 \tilde{x}$ and $\hat{y}=2 \tilde{y}$. The full emittances are $\hat{\epsilon}_{x, y}=4 \epsilon_{x, y}$, since we also have $\hat{p}_{x, y}=2 \sqrt{\left\langle\left(p_{x, y}-\left\langle p_{x, y}\right\rangle\right)^{2}\right\rangle}$. The envelope equation becomes

$$
\begin{aligned}
& \hat{x}^{\prime \prime}+K_{x}(s) \hat{x}-\frac{\hat{\epsilon}_{x}^{2}}{\hat{x}^{3}}-\frac{4 r_{0} \lambda}{\gamma^{3} \beta^{2}} \frac{1}{\hat{x}+\hat{y}}=0, \\
& \hat{y}^{\prime \prime}+K_{y}(s) \hat{y}-\frac{\hat{\epsilon}_{y}^{2}}{\hat{y}^{3}}-\frac{4 r_{0} \lambda}{\gamma^{3} \beta^{2}} \frac{1}{\hat{x}+\hat{y}}=0,
\end{aligned}
$$

which is the Kapchinsky-Vladimirsky envelope equation [8].

Now let us come back to Eq. (3.31), which is the more general envelope equation. Using the relations

$$
\frac{\beta_{x} \beta_{x}^{\prime \prime}}{2}+\frac{\beta_{x}^{\prime 2}}{4}+\beta_{x}^{2} K_{x}=1 \quad \text { and } \quad \frac{\beta_{y} \beta_{y}^{\prime \prime}}{2}+\frac{\beta_{y}^{\prime 2}}{4}+\beta_{y}^{2} K_{y}=1
$$

the substitutions

$$
\tilde{X}=\frac{\tilde{x}}{\sqrt{\epsilon_{x} \beta_{x}}} \quad \text { and } \quad \tilde{Y}=\frac{\tilde{y}}{\sqrt{\epsilon_{y} \beta_{y}}}
$$


and the Floquet phases

$$
\psi_{x}=\int_{0}^{s} \frac{d s}{\nu_{0 x} \beta_{x}} \text { and } \psi_{y}=\int_{0}^{s} \frac{d s}{\nu_{0 y} \beta_{y}},
$$

the envelope equation for the rms transverse beam spreads is transformed to

$$
\begin{aligned}
& \frac{d^{2} \tilde{X}}{d \psi_{x}^{2}}+\left(\nu_{0 x}^{2}+2 \nu_{0 x} \Delta \nu_{s x} \cos n_{x} \psi_{x}\right) \tilde{X}-\frac{\nu_{0 x}^{2}}{\tilde{X}^{3}}-\nu_{0 x} \Delta \nu_{0 x}^{\mathrm{spch}} \frac{\sigma_{x}+\sigma_{y}}{\sigma_{x} \tilde{X}+\sigma_{y} \tilde{Y}}=0, \\
& \frac{d^{2} \tilde{Y}}{d \psi_{y}^{2}}+\left(\nu_{0 y}^{2}+2 \nu_{0 y} \Delta \nu_{s y} \cos n_{y} \psi_{y}\right) \tilde{Y}-\frac{\nu_{0 y}^{2}}{\tilde{Y}^{3}}-\nu_{0 y} \Delta \nu_{0 y}^{\mathrm{spch}} \frac{\sigma_{x}+\sigma_{y}}{\sigma_{x} \tilde{X}+\sigma_{y} \tilde{Y}}=0 .
\end{aligned}
$$

In above, $\sigma_{x}=\sqrt{\epsilon_{x} \beta_{x}}$ and $\sigma_{y}=\sqrt{\epsilon_{y} \beta_{y}}$ are the rms spreads of the beam, and

$$
\Delta \nu_{x}^{\text {spch }}=\frac{\lambda r_{0} R^{2}}{\gamma^{3} \beta^{2} \nu_{0 x} \sigma_{x}\left(\sigma_{x}+\sigma_{y}\right)} \quad \text { and } \quad \Delta \nu_{y}^{\text {spch }}=\frac{\lambda r_{0} R^{2}}{\gamma^{3} \beta^{2} \nu_{0 y} \sigma_{y}\left(\sigma_{x}+\sigma_{y}\right)}
$$

are the maximum incoherent space-charge tune shifts at the center of the beam, with $\lambda=$ $N_{b} /(2 \pi R)$ being the average linear particle density. We have also included the parts in $K_{x}(s)$ and $K_{y}(s)$ that correspond to quadrupole gradient errors as forces possessing horizontal $n_{x}$-th harmonic and vertical $n_{y}$-th harmonic and total stopband widths $\Delta \nu_{s x}$ and $\Delta \nu_{s y}$.

We first solve for the static beam radii

$$
\tilde{X}=1+\xi_{x} \quad \text { and } \quad \tilde{Y}=1+\xi_{y}
$$

in terms of the incoherent space-charge tune shifts $\Delta \nu_{x}^{\text {spch }}$ and $\Delta \nu_{y}^{\mathrm{spch}}$ via the two parameters

$$
\Delta_{x}=\frac{\Delta \nu_{x}^{\mathrm{spch}}}{\nu_{0 x}} \text { and } \Delta_{y}=\frac{\Delta \nu_{y}^{\mathrm{spch}}}{\nu_{0 y}}
$$

Up to the second order, we get

$$
\xi_{x}=\frac{\Delta_{x}}{4}+\frac{\Delta_{x}^{2}}{16}-\frac{\Delta_{x} \Delta_{y}}{32} \quad \text { and } \quad \xi_{y}=\frac{\Delta_{y}}{4}+\frac{\Delta_{y}^{2}}{16}-\frac{\Delta_{x} \Delta_{y}}{32} .
$$

The change in static radii of the beam is due to the influence of the space-charge force. Next, the infinitesimal time-dependent displacements $\delta_{x}$ and $\delta_{y}$ are included:

$$
\tilde{X}=1+\xi_{x}+\delta_{x} \quad \text { and } \quad \tilde{Y}=1+\xi_{y}+\delta_{y} .
$$

Let us first study the special case of a round beam with $\sigma_{x}=\sigma_{y}$. However, the incoherent space-charge tune shifts can still be unequal for the horizontal and vertical because horizontal 
and vertical betatron tunes can be different. We obtain the equations for small-amplitude oscillations:

$$
\left(\begin{array}{c}
\frac{d^{2} \delta_{x}}{d \psi_{x}^{2}} \\
\frac{d^{2} \delta_{y}}{d \psi_{y}^{2}}
\end{array}\right)+\mathcal{M}\left(\begin{array}{c}
\delta_{x} \\
\delta_{y}
\end{array}\right)=\left(\begin{array}{c}
\nu_{0 x}^{2} \Delta \nu_{x}^{\mathrm{spch}} \cos n_{x} \psi_{x}+\mathcal{O}\left[\left(\Delta \nu_{x, y}^{\mathrm{spch}}\right)^{2}\right] \\
\nu_{0 y}^{2} \Delta \nu_{y}^{\mathrm{spch}} \cos n_{y} \psi_{y}+\mathcal{O}\left[\left(\Delta \nu_{x, y}^{\mathrm{spch}}\right)^{2}\right]
\end{array}\right),
$$

where

$$
\mathcal{M}=\left(\begin{array}{cc}
\left(4-\frac{5 \Delta_{x}}{2}-\frac{7 \Delta_{x}^{2}}{8}+\frac{\Delta_{x} \Delta_{y}}{4}\right) \nu_{0 x}^{2} & \left(\frac{\Delta_{x}}{2}-\frac{\Delta_{x}^{2}}{8}-\frac{\Delta_{x} \Delta_{y}}{8}\right) \nu_{0 x}^{2} \\
\left(\frac{\Delta_{y}}{2}-\frac{\Delta_{y}^{2}}{8}-\frac{\Delta_{x} \Delta_{y}}{8}\right) \nu_{0 y}^{2} & \left(4-\frac{5 \Delta_{y}}{2}-\frac{7 \Delta_{y}^{2}}{8}+\frac{\Delta_{x} \Delta_{y}}{4}\right) \nu_{0 y}^{2}
\end{array}\right),
$$

or

$$
\begin{gathered}
\mathcal{M}=\left(\begin{array}{c}
4 \nu_{0 x}^{2}-\frac{5 \nu_{0 x} \Delta \nu_{x}^{\mathrm{spch}}}{2}-\frac{7\left(\Delta \nu_{x}^{\mathrm{spch}}\right)^{2}}{8}+\frac{\nu_{0 x} \nu_{0 y} \Delta \nu_{x}^{\mathrm{spch}} \Delta \nu_{y}^{\mathrm{spch}}}{4} \\
\frac{\nu_{0 y} \Delta \nu_{y}^{\mathrm{spch}}}{2}-\frac{\left(\Delta \nu_{y}^{\mathrm{spch}}\right)^{2}}{8}-\frac{\nu_{0 x} \nu_{0 y} \Delta \nu_{x}^{\mathrm{spch}} \Delta \nu_{y}^{\mathrm{spch}}}{8} \\
\frac{\nu_{0 x} \Delta \nu_{x}^{\mathrm{spch}}}{2}-\frac{\left(\Delta \nu_{x}^{\mathrm{spch}}\right)^{2}}{8}-\frac{\nu_{0 x} \nu_{0 y} \Delta \nu_{x}^{\mathrm{spch}} \Delta \nu_{y}^{\mathrm{spch}}}{8} \\
4 \nu_{0 y}^{2}-\frac{5 \nu_{0 y} \Delta \nu_{y}^{\mathrm{spch}}}{2}-\frac{7\left(\Delta \nu_{y}^{\mathrm{spch}}\right)^{2}}{8}+\frac{\nu_{0 x} \nu_{0 y} \Delta \nu_{x}^{\mathrm{spch}} \Delta \nu_{y}^{\mathrm{spch}}}{4}
\end{array}\right) .
\end{gathered}
$$

It is clear that Eq. (3.43) is just a set of driven oscillatory equations. Thus the eigentunes of $\mathcal{M}$ will give the coherent tunes of oscillation of the beam envelope. Analytic expressions for the coherent eigentunes are straight forward, although pretty messy. Fortunately, we can resort to numerical solution. The coherent eigentunes $\nu_{\text {coh }}$ are obtained by just solving the quadratic equation

$$
\nu_{\text {coh }}^{4}-\nu_{\text {coh }}^{2}\left(\mathcal{M}_{11}+\mathcal{M}_{22}\right)+\mathcal{M}_{11} \mathcal{M}_{22}-\mathcal{M}_{12} \mathcal{M}_{21}=0
$$

where $\mathcal{M}_{i j}$ denote the matrix elements of $\mathcal{M}$. There are only two physical eigentunes which are real and positive. For beam rotation in the CR, the betatron tunes are $\nu_{0 x}=6.76$ and $\nu_{0 y}=8.44$. The maximum incoherent space-charge tune shifts given by Ref. [1] are $\Delta \nu_{x}^{\text {spch }}=0.14$ and $\Delta \nu_{y}^{\text {spch }}=0.16$. The two coherent eigentunes are $\nu_{1 \text { coh }}=2 \times 6.713$ and $\nu_{2 \text { coh }}=2 \times 8.393$. Thus the two coherent space-charge tune shifts are $\Delta \nu_{1 \text { coh }}^{\text {spch }}=2 \times 0.047$ and $\Delta \nu_{2 \text { coh }}^{\text {spch }}=2 \times 0.047$. 
If we include only first-order incoherent space-charge tune shifts in $\mathcal{M}$, these coherent tune shifts turn out to be the same within three significant figures. Thus the first-order equation would be sufficient, although the two incoherent space-charge tune shifts $\Delta \nu_{x}^{\text {spch }}=$ 0.14 and $\Delta \nu_{y}^{\text {spch }}=0.16$, both may not be too small. In that case the solution can be written in a more manageable form:

$$
\begin{aligned}
\nu_{\text {coh }}^{2}=2( & \left.\nu_{0 x}^{2}+\nu_{0 y}^{2}\right)-\frac{5}{4}\left(\nu_{0 x} \Delta \nu_{x}^{\text {spch }}+\nu_{0 y} \Delta \nu_{y}^{\text {spch }}\right) \\
\pm & \left\{4\left(\nu_{0 x}^{2}-\nu_{0 y}^{2}\right)^{2}-5\left(\nu_{0 x}^{2}-\nu_{0 y}^{2}\right)\left(\nu_{0 x} \Delta \nu_{x}^{\text {spch }}+\nu_{0 y} \Delta \nu_{y}^{\text {spch }}\right)\right. \\
& \left.+\frac{25}{16}\left(\nu_{0 x} \Delta \nu_{x}^{\text {spch }}-\nu_{0 y} \Delta \nu_{y}^{\text {spch }}\right)^{2}+\frac{1}{4} \nu_{0 x} \nu_{0 y} \Delta \nu_{x}^{\text {spch }} \Delta \nu_{y}^{\text {spch }}\right\}^{1 / 2} .
\end{aligned}
$$

In addition, when the incoherent space-charge tune shifts are equal in the two transverse directions, $\Delta \nu_{x}^{\text {spch }}=\Delta \nu_{y}^{\text {spch }}$, the above reduces to

$$
\begin{aligned}
\nu_{\text {coh }}^{2}= & 2\left(\nu_{0 x}^{2}+\nu_{0 y}^{2}\right)-\frac{5}{4}\left(\nu_{0 x}+\nu_{0 y}\right) \Delta \nu_{x}^{\mathrm{spch}} \\
& \pm \sqrt{4\left(\nu_{0 x}^{2}-\nu_{0 y}^{2}\right)^{2}-5\left(\nu_{0 x}-\nu_{0 y}\right)^{2}\left(\nu_{0 x}+\nu_{0 y}\right) \Delta \nu_{x}^{\mathrm{spch}}+\left[\frac{25}{16}\left(\nu_{0 x}-\nu_{0 y}\right)^{2}+\frac{1}{4} \nu_{0 x} \nu_{0 y}\right]\left(\Delta \nu_{x}^{\mathrm{spch}}\right)^{2}} .
\end{aligned}
$$

Of course, one may argue that terms involving second order in incoherent space-charge tune shifts should be omitted in Eqs. (3.47) and (3.48) because only terms with first order space-charge tune shift are included in $\mathcal{M}$. When the two bare tunes are close so that $\left|\nu_{0 x}-\nu_{0 y}\right| \ll \nu_{0 x} \Delta \nu_{x}^{\text {spch }}$, the two coherent tunes are

$$
\nu_{\mathrm{coh}}^{2}=\left\{\begin{array} { l } 
{ 4 \overline { \nu } ^ { 2 } - 2 \nu _ { 0 x } \Delta \nu _ { x } ^ { \mathrm { spch } } } \\
{ 4 \overline { \nu } ^ { 2 } - 3 \nu _ { 0 y } \Delta \nu _ { x } ^ { \mathrm { spch } } }
\end{array} \quad \text { or } \quad \nu _ { \mathrm { coh } } \approx \left\{\begin{array}{l}
2\left(\bar{\nu}-\frac{1}{4} \Delta \nu_{x}^{\mathrm{spch}}\right) \\
2\left(\bar{\nu}-\frac{3}{8} \Delta \nu_{x}^{\mathrm{spch}}\right)
\end{array}\right.\right.
$$

where $2 \bar{\nu}^{2}=\nu_{0 x}^{2}+\nu_{0 y}^{2}$. This represents that the two transverse directions are tightly coupled. The eigenfunctions are $\sim\left(\delta_{x}+\delta_{y}\right)$ for the upper solution and $\sim\left(\delta_{x}-\delta_{y}\right)$ for the lower solution. Thus, the upper solution is the symmetric breathing mode where the oscillations are in phase in both transverse directions and the tune is $2\left(\bar{\nu}-\frac{1}{4} \Delta \nu_{x}^{\text {spch }}\right)$. The lower solution is the antisymmetric mode where the beam envelope oscillates out of phase in the two transverse directions with tune $2\left(\bar{\nu}-\frac{3}{8} \Delta \nu_{x}^{\text {spch }}\right)$.

If the tune split is large so that $\left|\nu_{0 x}-\nu_{0 y}\right| \gg \nu_{0 x} \Delta \nu_{x}^{\text {spch }}$, the oscillations in the two transverse directions are almost uncoupled. The envelope oscillations in the two transverse 
directions are just two independent oscillators. The two coherent tunes becomes

$$
\nu_{\mathrm{coh}}^{2}=\left\{\begin{array} { l } 
{ 4 \nu _ { 0 x } ^ { 2 } - \frac { 5 } { 2 } \nu _ { 0 x } \Delta \nu _ { x } ^ { \mathrm { spch } } } \\
{ 4 \nu _ { 0 y } ^ { 2 } - \frac { 5 } { 2 } \nu _ { 0 y } \Delta \nu _ { y } ^ { \mathrm { spch } } }
\end{array} \quad \text { or } \quad \nu _ { \mathrm { coh } } \approx \left\{\begin{array}{l}
2\left(\nu_{0 x}-\frac{5}{16} \Delta \nu_{x}^{\mathrm{spch}}\right), \\
2\left(\nu_{0 y}-\frac{5}{16} \Delta \nu_{y}^{\mathrm{spch}}\right) .
\end{array}\right.\right.
$$

\subsection{General Solution}

The most general situation is when $\Delta \nu_{x}^{\text {spch }} \neq \Delta \nu_{y}^{\text {spch }}$ and $\sigma_{x} \neq \sigma_{y}$. First we substitute the static solution of Eq. (3.39) into the envelope equation (3.37). The solution up to second order in space-charge tune shifts is

$$
\begin{aligned}
& \xi_{x}=\frac{\Delta_{x}}{4}+\frac{1+3 \sigma_{y} / \sigma_{x}}{1+\sigma_{y} / \sigma_{x}} \frac{\Delta_{x}^{2}}{32}-\frac{\sigma_{y} / \sigma_{x}}{1+\sigma_{y} / \sigma_{x}} \frac{\Delta_{x} \Delta_{y}}{16}, \\
& \xi_{y}=\frac{\Delta_{y}}{4}+\frac{1+3 \sigma_{x} / \sigma_{y}}{1+\sigma_{x} / \sigma_{y}} \frac{\Delta_{y}^{2}}{32}-\frac{\sigma_{x} / \sigma_{y}}{1+\sigma_{x} / \sigma_{y}} \frac{\Delta_{x} \Delta_{y}}{16} .
\end{aligned}
$$

Now the oscillatory parts of the solution, $\delta_{x}$ and $\delta_{y}$, are added. The result is the driven oscillatory equation (3.43), but with the oscillatory matrix $\mathcal{M}$ replaced by

$$
\begin{aligned}
& \frac{\mathcal{M}_{11}}{\nu_{0 x}^{2}}=4-\frac{2+\frac{3 \sigma_{y}}{\sigma_{x}}}{1+\frac{\sigma_{y}}{\sigma_{x}}} \Delta_{x}-\frac{7+\frac{12 \sigma_{y}}{\sigma_{x}}+\left(\frac{3 \sigma_{y}}{\sigma_{x}}\right)^{2}}{1+\frac{\sigma_{y}}{\sigma_{x}}} \frac{\Delta_{x}^{2}}{8}+\frac{\frac{\sigma_{y}}{\sigma_{x}}\left(1+\frac{3 \sigma_{y}}{\sigma_{x}}\right)}{\left(1+\frac{\sigma_{y}}{\sigma_{x}}\right)^{2}} \frac{\Delta_{x} \Delta_{y}}{4} \\
& \frac{\mathcal{M}_{12}}{\nu_{0 x}^{2}}=\frac{\frac{\sigma_{y}}{\sigma_{x}}}{1+\frac{\sigma_{y}}{\sigma_{x}}} \Delta_{x}-\frac{\frac{\sigma_{y}}{\sigma_{x}}}{\left(1+\frac{\sigma_{y}}{\sigma_{x}}\right)^{2}} \frac{\Delta_{x}^{2}}{2}-\frac{\left(\frac{\sigma_{y}}{\sigma_{x}}\right)^{2}}{\left(1+\frac{\sigma_{y}}{\sigma_{x}}\right)^{2}} \frac{\Delta_{x} \Delta_{y}}{2} \\
& \frac{\mathcal{M}_{21}}{\nu_{0 y}^{2}}=\frac{\frac{\sigma_{x}}{\sigma_{y}}}{1+\frac{\sigma_{x}}{\sigma_{y}}} \Delta_{y}-\frac{\frac{\sigma_{x}}{\sigma_{y}}}{\left(1+\frac{\sigma_{x}}{\sigma_{y}}\right)^{2}} \frac{\Delta_{y}^{2}}{2}-\frac{\left(\frac{\sigma_{x}}{\sigma_{y}}\right)^{2}}{\left(1+\frac{\sigma_{x}}{\sigma_{y}}\right)^{2}} \frac{\Delta_{x} \Delta_{y}}{2} \\
& \frac{\mathcal{M}_{22}}{\nu_{0 y}^{2}}=4-\frac{2+\frac{3 \sigma_{x}}{\sigma_{y}}}{1+\frac{\sigma_{x}}{\sigma_{y}}} \Delta_{y}-\frac{7+\frac{12 \sigma_{x}}{\sigma_{y}}+\left(\frac{3 \sigma_{x}}{\sigma_{y}}\right)^{2}}{1+\frac{\sigma_{x}}{\sigma_{y}}} \frac{\Delta_{y}^{2}}{8}+\frac{\frac{\sigma_{x}}{\sigma_{y}}\left(1+\frac{3 \sigma_{x}}{\sigma_{y}}\right)}{\left(1+\frac{\sigma_{x}}{\sigma_{y}}\right)^{2}} \frac{\Delta_{x} \Delta_{y}}{4} .
\end{aligned}
$$

We now substitute the beam radii $\sigma_{x}=6.464 \mathrm{~mm}$ and $\sigma_{y}=5.391 \mathrm{~mm}$ at turn 660 , but still employ the incoherent space-charge tune shifts $\Delta \nu_{x}^{\text {spch }}=0.14$ and $\Delta \nu_{y}^{\text {spch }}=0.16$ quoted in Ref. [1]. The coherent eigentunes are $\nu_{1 \mathrm{coh}}=2 \times 6.717$ and $\nu_{2 \mathrm{coh}}=2 \times 8.389$. The quadrupole coherent tune shifts are $\Delta \nu_{1 \text { coh }}=2 \times 0.043$ and $\Delta \nu_{2 \text { coh }}=2 \times 0.051$.

If we substitute instead the incoherent space-charge tune shifts obtained from our beam rotation computation, i.e., $\Delta \nu_{x}^{\text {spch }}=0.190$ and $\Delta \nu_{y}^{\text {spch }}=0.183$, the eigentunes become $\nu_{1 \text { coh }}=2 \times 6.701$ and $\nu_{2 \text { coh }}=2 \times 8.382$. and the coherent tune shift become $\Delta \nu_{1 \text { coh }}^{\text {spch }}=2 \times 0.059$ and $\Delta \nu_{2 \text { coh }}^{\text {spch }}=2 \times 0.058$. 


\subsection{Comments}

1. Our computation shows that the quadrupole coherent space-charge tune shifts are of the order of $\Delta \nu_{1,2 \text { coh }}^{\text {spch }}=2 \times 0.060$, less than one half of the incoherent tune shifts. Since the bare tunes are $\nu_{0 x}=6.76$ and $\nu_{0 y}=8.44$, the shifts are far away from the half-integer resonances.

2. The computation has been for the center slice of the beam. If each transverse slice of the beam is independent and is treated at a coasting beam, the quadrupole coherent space-charge tune shifts for these off-center slices will be smaller because the local beam current will be smaller. In other words the coherent tune shifts become coherent tune spreads. Nevertheless these spreads will still be far away from the half-integer resonances.

However, each transverse slice is not independent because of synchrotron motion. The correct way to attack the problem will be some three-dimensional theory that includes the longitudinal as well, so that the coherent oscillation frequencies correspond to collective modes of the bunch as a whole. The solution of such a problem will be left to a future article.

\section{Conclusions}

We have studied the space-charge effects on the bunch narrowing rotation in the CR, both longitudinally and transversely. Our investigation shows that at the present bunch intensity the longitudinal space-charge force, although not too small, is still no able to cause significant disastrous effects, like big bunch-width increase, to the beam rotation. The transverse spacecharge force will drive coherent quadrupole breathing modes of oscillation in the beam. But at the present beam intensity, these coherent frequency shifts, $\Delta \nu_{1,2 \text { coh }}^{\text {spch }} \sim 2 \times 0.06$ will not be large enough to lead these modes into the half-integer stopbands. However, if the bunch intensity is doubled in the future, one will see significant beam-profile distortion in the beam rotation as depicted in Fig. 4. Transversely, the coherent quadrupole tune shifts are still not large enough to shift the beam into the half-integer stopbands even if the bunch intensity is doubled. But the encountering of other higher-order parametric resonances can be possible. 


\section{References}

[1] Y. Alexahin and D. Neuffer, Design of Accumulator and Compressor Rings for the Project-X Based Proton Driver, Proceedings of IPAC2012, May 20-35, 2012, New Orleans, Louisiana, USA, p.1260, 2012.

[2] J. Wei, Longitudinal Dynamics of the Non-Adiabatic Regime on Alternating-Gradient Synchrotrons, Thesis, State University of New York at stony Brook, May 1990.

[3] R. Baartman, Betatron Resonances with Space Charge, Proceedings of Int. Workshop on Emittance in Circular Accelerators Nov. 1994, KEK, Japan, KEK report 95-7, p.273; R. Baartman, Betatron Resonances with Space Charge, Proceedings of Workshop on Space Charge Physics in High Intensity Hadron Rings, p.73, Ed. Luccio, A.U., and Weng, W.T., (Shelter Island, New York, May 4-7, 1998).

[4] S. Machida, Space Charge Effects in Low Energy Proton Synchrotrons, Nucl. Inst. Meth. A309, 43 (1991).

[5] S. Machida and M. Ikegami, Simulation of Space-Charge Effects in a Synchrotron, Proceedings of Workshop on Space Charge Physics in High Intensity Hadron Rings, p.73, Ed. Luccio, A.U., and Weng, W.T., (Shelter Island, New York, May 4-7, 1998).

[6] F. Sacherer, RMS Envelope Equations with Space Charge, IEEE Trans. Nucl. Sci. NS18, 1105 (1971). See also the longer report of the same title in CERN Report CERNSI-Int.-DL/70-12, Nov., 1970.

[7] F. J. Sacherer, Transverse Space-Charge Effects in Circular Accelerators, Thesis, University of California, Berkeley, Report UCRL-18454, 1968.

[8] I. M. Kapchinsky and V. V. Vladimirsky, Limitations of Proton Beam Current in a Strong Focusing Linear Accelerator Associated with the Beam Space Charge, Proc. Int. Conf. on High Energy Accel., ed. L. Kowarski (Geneva, Sept. 14-19, 1959), p. 274. 